\title{
Reduction Formulae for Products of Theta Functions
}

\author{
P. L. Walker \\ 7 Redgrove Park, \\ Cheltenham, \\ GL51 6QY \\ UK
}

pandjw@hotmail.co.uk

In four cases it is already known that the product of two distinct Jacobian theta functions having the same variable $z$ and the same nome $q$ is a multiple of a single Jacobian theta function, with the multiple independent of $z$. The main purpose of the present note is to show that this property also applies in the remaining two cases.

Key words: Jacobian theta functions, especially products; normalizing factors.

Accepted: September 19, 2012

Published: November 6, 2012

http://dx.doi.org/10.6028/jres.117.017

\section{Introduction}

The formulae

$$
\begin{gathered}
\frac{\theta_{1}(z, q) \theta_{2}(z, q)}{\theta_{1}\left(2 z, q^{2}\right)}=\frac{\theta_{3}(z, q) \theta_{4}(z, q)}{\theta_{4}\left(2 z, q^{2}\right)}=\theta_{4}\left(0, q^{2}\right), \\
\frac{\theta_{1}\left(z, q^{2}\right) \theta_{4}\left(z, q^{2}\right)}{\theta_{1}(z, q)}=\frac{\theta_{2}\left(z, q^{2}\right) \theta_{3}\left(z, q^{2}\right)}{\theta_{2}(z, q)}=\frac{1}{2} \theta_{2}(0, q),
\end{gathered}
$$

occur in the theory of Jacobian theta functions and their applications to Jacobian elliptic functions. For example, Eq. (1) leads to the Landen transformations (Ref. [1], Subsection 20.7(vi) and Section 22.7).

The main purpose of the present paper is to prove that

$$
\frac{\theta_{1}\left(z, q^{2}\right) \theta_{3}\left(z, q^{2}\right)}{\theta_{1}(z, i q)}=\frac{\theta_{2}\left(z, q^{2}\right) \theta_{4}\left(z, q^{2}\right)}{\theta_{2}(z, i q)}=i^{-1 / 4} \sqrt{\frac{\theta_{2}\left(0, q^{2}\right) \theta_{4}\left(0, q^{2}\right)}{2}}
$$

thereby covering the remaining two cases.

However, we shall also prove Eqs. (1) and (2) by the same method, thereby providing new proofs for these equations. Incidentally, since the right-hand sides of Eqs. (1), (2), and (3) are independent of $z$, we shall refer to them as normalizing factors. 


\section{Infinite Products}

We begin with the well-known expansions of the theta functions as infinite products (Ref. [1], Eqs. (20.5.1)-(20.5.4)):

$$
\begin{aligned}
& \theta_{1}(z, q)=2 q^{1 / 4} \sin z \prod_{n=1}^{\infty}\left(1-q^{2 n}\right)\left(1-2 q^{2 n} \cos (2 z)+q^{4 n}\right), \\
& \theta_{2}(z, q)=2 q^{1 / 4} \cos z \prod_{n=1}^{\infty}\left(1-q^{2 n}\right)\left(1+2 q^{2 n} \cos (2 z)+q^{4 n}\right), \\
& \theta_{3}(z, q)=\prod_{n=1}^{\infty}\left(1-q^{2 n}\right)\left(1+2 q^{2 n-1} \cos (2 z)+q^{4 n-2}\right), \\
& \theta_{4}(z, q)=\prod_{n=1}^{\infty}\left(1-q^{2 n}\right)\left(1-2 q^{2 n-1} \cos (2 z)+q^{4 n-2}\right) .
\end{aligned}
$$

In particular,

$$
\begin{aligned}
& \theta_{1}^{\prime}(0, q)=2 q^{1 / 4} \prod_{n=1}^{\infty}\left(1-q^{2 n}\right)^{3} \\
& \theta_{2}(0, q)=2 q^{1 / 4} \prod_{n=1}^{\infty}\left(1-q^{2 n}\right)\left(1+q^{2 n}\right)^{2} \\
& \theta_{3}(0, q)=\prod_{n=1}^{\infty}\left(1-q^{2 n}\right)\left(1+q^{2 n-1}\right)^{2} \\
& \theta_{4}(0, q)=\prod_{n=1}^{\infty}\left(1-q^{2 n}\right)\left(1-q^{2 n-1}\right)^{2}
\end{aligned}
$$

The prime in $\theta_{1}^{\prime}(0, q)$ indicates differentiation with respect to the variable $z$. In these formulae, $z, q$ are complex and $|q|<1$ to ensure convergence. Here $q^{1 / 4}$ denotes the principal value of the root. With $q=e^{i \pi \tau}$, $\tau$ is a complex parameter with positive imaginary part.

With this notation we have

$$
\begin{aligned}
1-2 q^{2 n} \cos (2 z)+q^{4 n} & =1-2 e^{2 n i \pi \tau} \cos (2 z)+e^{4 n i \pi \tau} \\
& =2 e^{2 n i \pi \tau}(\cos (2 n \pi \tau)-\cos (2 z)) \\
& =4 e^{2 n i \pi \tau} \sin (z-n \pi \tau) \sin (z+n \pi \tau),
\end{aligned}
$$

and hence

$$
\frac{\theta_{1}(z, q)}{\theta_{1}^{\prime}(0, q)}=\sin z \prod_{n=1}^{\infty} \frac{\sin (n \pi \tau-z) \sin (n \pi \tau+z)}{\sin ^{2}(n \pi \tau)}
$$

Note that as $n \rightarrow \infty$, the $n$th factor in this infinite product is $1+O\left(e^{2 n i \pi \tau}\right)$, which ensures rapid convergence since $\mathfrak{I} \tau>0$.

Repetition of this calculation (details omitted) for $\theta_{2}, \theta_{3}$, and $\theta_{4}$ yields

$$
\frac{\theta_{2}(z, q)}{\theta_{2}(0, q)}=\cos z \prod_{n=1}^{\infty} \frac{\cos (n \pi \tau-z) \cos (n \pi \tau+z)}{\cos ^{2}(n \pi \tau)}
$$




$$
\frac{\theta_{3}(z, q)}{\theta_{3}(0, q)}=\prod_{n=1}^{\infty} \frac{\cos \left(\left(n-\frac{1}{2}\right) \pi \tau-z\right) \cos \left(\left(n-\frac{1}{2}\right) \pi \tau+z\right)}{\cos ^{2}\left(\left(n-\frac{1}{2}\right) \pi \tau\right)}
$$

and

$$
\frac{\theta_{4}(z, q)}{\theta_{4}(0, q)}=\prod_{n=1}^{\infty} \frac{\sin \left(\left(n-\frac{1}{2}\right) \pi \tau-z\right) \sin \left(\left(n-\frac{1}{2}\right) \pi \tau+z\right)}{\sin ^{2}\left(\left(n-\frac{1}{2}\right) \pi \tau\right)}
$$

The required results now follow on combining these equations in pairs.

For the product $\theta_{1} \theta_{2}$ we have from Eqs. (7) and (8)

$$
\begin{aligned}
\frac{\theta_{1}(z, q)}{\theta_{1}^{\prime}(0, q)} \frac{\theta_{2}(z, q)}{\theta_{2}(0, q)}= & \sin z \cos z \prod_{n=1}^{\infty} \frac{\sin (n \pi \tau-z) \sin (n \pi \tau+z)}{\sin ^{2}(n \pi \tau)} \\
& \times \frac{\cos (n \pi \tau-z) \cos (n \pi \tau+z)}{\cos ^{2}(n \pi \tau)} \\
= & \frac{1}{2} \sin (2 z) \prod_{n=1}^{\infty} \frac{\sin (2 n \pi \tau-2 z) \sin (2 n \pi \tau+2 z)}{\sin ^{2}(2 n \pi \tau)} \\
= & \frac{1}{2} \frac{\theta_{1}\left(2 z, q^{2}\right)}{\theta_{1}^{\prime}\left(0, q^{2}\right)},
\end{aligned}
$$

where we have used the identity $q^{2}=e^{2 i \pi \tau}$. Hence

$$
\frac{\theta_{1}(z, q) \theta_{2}(z, q)}{\theta_{1}\left(2 z, q^{2}\right)}=\frac{1}{2} \frac{\theta_{1}^{\prime}(0, q) \theta_{2}(0, q)}{\theta_{1}^{\prime}\left(0, q^{2}\right)}
$$

The translation $z \rightarrow z+\frac{1}{2} \tau$ changes $\theta_{1} \rightarrow \theta_{3}$ and $\theta_{2} \rightarrow \theta_{4}$. Hence we have

$$
\frac{\theta_{1}(z, q) \theta_{2}(z, q)}{\theta_{1}\left(2 z, q^{2}\right)}=\frac{\theta_{3}(z, q) \theta_{4}(z, q)}{\theta_{3}\left(2 z, q^{2}\right)}=\frac{1}{2} \frac{\theta_{1}^{\prime}(0, q) \theta_{2}(0, q)}{\theta_{1}^{\prime}\left(0, q^{2}\right)}
$$

which is the first required result, Eq. (1), except for the normalizing factor. We will return to this below and for now concentrate on the quotients of functions of $z$.

Turning to the product $\theta_{1} \theta_{4}$, we have from Eqs. (7) and (10) 


$$
\begin{aligned}
\frac{\theta_{1}\left(z, q^{2}\right)}{\theta_{1}^{\prime}\left(0, q^{2}\right)} \frac{\theta_{4}\left(z, q^{2}\right)}{\theta_{4}\left(0, q^{2}\right)}= & \sin z \prod_{n=1}^{\infty} \frac{\sin (2 n \pi \tau-z) \sin (2 n \pi \tau+z)}{\sin ^{2}(2 n \pi \tau)} \\
& \times \frac{\sin ((2 n-1) \pi \tau-z) \sin ((2 n-1) \pi \tau+z)}{\sin ^{2}((2 n-1) \pi \tau)} \\
= & \sin z \prod_{n=1}^{\infty} \frac{\sin (n \pi \tau-z) \sin (n \pi \tau+z)}{\sin ^{2}(n \pi \tau)}=\frac{\theta_{1}(z, q)}{\theta_{1}^{\prime}(0, q)}
\end{aligned}
$$

where in the last line we have combined the even $(2 n)$ and the odd $(2 n-1)$ terms to obtain the complete sequence. Hence on rearranging we have,

$$
\frac{\theta_{1}\left(z, q^{2}\right) \theta_{4}\left(z, q^{2}\right)}{\theta_{1}(z, q)}=\frac{\theta_{1}^{\prime}\left(0, q^{2}\right) \theta_{4}\left(0, q^{2}\right)}{\theta_{1}^{\prime}(0, q)},
$$

followed by translation in $z$ by $\frac{1}{2} \pi$ :

$$
\frac{\theta_{1}\left(z, q^{2}\right) \theta_{4}\left(z, q^{2}\right)}{\theta_{1}(z, q)}=\frac{\theta_{2}\left(z, q^{2}\right) \theta_{3}\left(z, q^{2}\right)}{\theta_{2}(z, q)}=\frac{\theta_{1}^{\prime}\left(0, q^{2}\right) \theta_{4}\left(0, q^{2}\right)}{\theta_{1}^{\prime}(0, q)},
$$

which is Eq. (2), except for the normalizing factor.

For the third identity we note that $q \rightarrow i q$ corresponds to $\tau \rightarrow \tau+\frac{1}{2}$. From Eqs. (7) and (9) we have

$$
\begin{aligned}
\frac{\theta_{1}\left(z, q^{2}\right) \theta_{3}\left(z, q^{2}\right)}{\theta_{1}^{\prime}\left(0, q^{2}\right) \theta_{3}\left(0, q^{2}\right)}= & \sin z \prod_{n=1}^{\infty} \frac{\sin (2 n \pi \tau-z) \sin (2 n \pi \tau+z)}{\sin ^{2}(2 n \pi \tau)} \\
& \times \frac{\cos ((2 n-1) \pi \tau-z) \cos ((2 n-1) \pi \tau+z)}{\cos ^{2}((2 n-1) \pi \tau)},
\end{aligned}
$$

and

$$
\frac{\theta_{1}(z, i q)}{\theta_{1}^{\prime}(0, i q)}=\sin z \prod_{n=1}^{\infty} \frac{\sin \left(n \pi\left(\tau+\frac{1}{2}\right)-z\right) \sin \left(n \pi\left(\tau+\frac{1}{2}\right)+z\right)}{\sin ^{2}\left(n \pi\left(\tau+\frac{1}{2}\right)\right)}
$$

Now in Eq. (18), we separate the terms according to the parity of $n$.

If $n=2 k(k \geq 1)$ is even, then

$$
\sin \left(n \pi\left(\tau+\frac{1}{2}\right) \pm z\right)=(-1)^{k} \sin (2 k \pi \tau \pm z)
$$

and

$$
\sin ^{2}\left(n \pi\left(\tau+\frac{1}{2}\right)\right)=\sin ^{2}(2 k \pi \tau)
$$


corresponding to the first quotient on the right-hand side of Eq. (17).

Similarly if $n=2 k-1 \quad(k \geq 1)$ is odd, then

$$
\begin{aligned}
\sin \left(n \pi\left(\tau+\frac{1}{2}\right) \pm z\right) & =\sin \left((2 k-1) \pi\left(\tau+\frac{1}{2}\right) \pm z\right) \\
& =(-1)^{k} \cos ((2 k-1) \pi \tau \pm z)
\end{aligned}
$$

and

$$
\sin ^{2}\left((2 k-1) \pi\left(\tau+\frac{1}{2}\right)\right)=\cos ^{2}((2 k-1) \pi \tau),
$$

corresponding to the second quotient in Eq. (17). Hence

$$
\frac{\theta_{1}\left(z, q^{2}\right) \theta_{3}\left(z, q^{2}\right)}{\theta_{1}^{\prime}\left(0, q^{2}\right) \theta_{3}\left(0, q^{2}\right)}=\frac{\theta_{1}(z, i q)}{\theta_{1}^{\prime}(0, i q)}
$$

or

$$
\frac{\theta_{1}\left(z, q^{2}\right) \theta_{3}\left(z, q^{2}\right)}{\theta_{1}(z, i q)}=\frac{\theta_{1}^{\prime}\left(0, q^{2}\right) \theta_{3}\left(0, q^{2}\right)}{\theta_{1}^{\prime}(0, i q)}
$$

and translation in $z$ by $\frac{1}{2} \pi$ gives

$$
\frac{\theta_{1}\left(z, q^{2}\right) \theta_{3}\left(z, q^{2}\right)}{\theta_{1}(z, i q)}=\frac{\theta_{2}\left(z, q^{2}\right) \theta_{4}\left(z, q^{2}\right)}{\theta_{2}(z, i q)}=\frac{\theta_{1}^{\prime}\left(0, q^{2}\right) \theta_{3}\left(0, q^{2}\right)}{\theta_{1}^{\prime}(0, i q)}
$$

which is Eq. (3) apart from the normalizing factor.

\section{Normalizing Factors}

If we compare our results (13), (16), and (25), with the desired Eqs. (1), (2), and (3), then we see that we have to verify the following identities:

$$
\begin{aligned}
& \frac{1}{2} \frac{\theta_{1}^{\prime}(0, q) \theta_{2}(0, q)}{\theta_{1}^{\prime}\left(0, q^{2}\right)}=\theta_{4}\left(0, q^{2}\right) \\
& \frac{\theta_{1}^{\prime}\left(0, q^{2}\right) \theta_{4}\left(0, q^{2}\right)}{\theta_{1}^{\prime}(0, q)}=\frac{1}{2} \theta_{2}(0, q),
\end{aligned}
$$

and

$$
\frac{\theta_{1}^{\prime}\left(0, q^{2}\right) \theta_{3}\left(0, q^{2}\right)}{\theta_{1}^{\prime}(0, i q)}=i^{-1 / 4} \sqrt{\frac{\theta_{2}\left(0, q^{2}\right) \theta_{4}\left(0, q^{2}\right)}{2}}
$$


The following identity, which goes back at least to Euler, is needed. When $|q|<1$ :

$$
\begin{aligned}
P(q) & =\prod_{n=1}^{\infty}\left(1+q^{n}\right)\left(1-q^{2 n-1}\right) \\
& =\{(1+q)(1-q)\}\left\{\left(1+q^{2}\right)\left(1-q^{3}\right)\right\}\left\{\left(1+q^{3}\right)\left(1-q^{5}\right)\right\} \cdots=1 .
\end{aligned}
$$

To verify this, we note that on expanding the product in powers of $q$, only a finite number of factors are needed to determine the coefficient of each power, so that no limiting process is involved and the terms may be re-ordered without affecting the value of the product. (Alternatively, for an analytic argument, the rearrangement is justified by absolute convergence.) Therefore

$$
\begin{aligned}
P(q)= & \left\{(1+q)\left(1+q^{2}\right)(1-q)\right\}\left\{\left(1+q^{3}\right)\left(1+q^{4}\right)\left(1-q^{3}\right)\right\} \\
& \times\left\{\left(1+q^{5}\right)\left(1+q^{6}\right)\left(1-q^{5}\right)\right\} \cdots \\
= & \left\{\left(1+q^{2}\right)\left(1-q^{2}\right)\right\}\left\{\left(1+q^{4}\right)\left(1-q^{6}\right)\right\}\left\{\left(1+q^{6}\right)\left(1-q^{10}\right)\right\} \cdots \\
= & \prod_{n=1}^{\infty}\left\{\left(1+q^{2 n}\right)\left(1-q^{4 n-2}\right)\right\}=P\left(q^{2}\right) .
\end{aligned}
$$

If $P(q)$ is not identically 1 , then there will be a least power of $q$, say $q^{k}$, whose coefficient is not zero. But then $q^{2 k}$ will be the least power of $q$ in $P\left(q^{2}\right)$ whose coefficient is not zero, so $P(q)$ and $P\left(q^{2}\right)$ cannot be equal, resulting in a contradiction.

To prove Eq. (26) we substitute from Eqs. (5), and see that we require

$$
\left(2 q^{1 / 4}\right)^{2} \prod_{n=1}^{\infty}\left(1-q^{2 n}\right)^{4}\left(1+q^{2 n}\right)^{2}=2\left(2 q^{1 / 2}\right) \prod_{n=1}^{\infty}\left(1-q^{4 n}\right)^{4}\left(1-q^{4 n-2}\right)^{2}
$$

After division by $4 q^{1 / 2} \prod_{n=1}^{\infty}\left(1-q^{2 n}\right)^{2}\left(1+q^{2 n}\right)$, we see that the last equation is equivalent to

$$
\prod_{n=1}^{\infty}\left(1-q^{2 n}\right)^{2}\left(1+q^{2 n}\right)=\prod_{n=1}^{\infty}\left(1-q^{4 n}\right)^{2}\left(1-q^{4 n-2}\right)
$$

Replacement of the term $\left(1-q^{4 n}\right)$ by $\left(1-q^{2 n}\right)\left(1+q^{2 n}\right)$, and use of Eq. (29) with $q^{2}$ in place of $q$, completes the proof.

For Eq. (27) we require similarly

$$
2 q^{1 / 2} \prod_{n=1}^{\infty}\left(1-q^{4 n}\right)^{4}\left(1-q^{4 n-2}\right)^{2}=\frac{\left(2 q^{1 / 4}\right)^{2}}{2} \prod_{n=1}^{\infty}\left(1-q^{2 n}\right)^{4}\left(1+q^{2 n}\right)^{2}
$$

or, on division by $2 q^{1 / 2} \prod_{n=1}^{\infty}\left(1-q^{4 n}\right)^{2}\left(1-q^{4 n-2}\right)$ :

$$
\prod_{n=1}^{\infty}\left(1-q^{4 n}\right)^{2}\left(1-q^{4 n-2}\right)=\prod_{n=1}^{\infty}\left(1-q^{2 n}\right)^{2}\left(1+q^{2 n}\right)
$$

This completes the proof of Eq. (27). 
Finally, for Eq. (28) we begin by calculating the asymptotic values of each side as $q \rightarrow 0$ through positive real values. On the left-hand side we have

$$
\left\{2\left(q^{2}\right)^{1 / 4} /\left(2(i q)^{1 / 4}\right)\right\}\left(1+O\left(q^{2}\right)\right)=(q / i)^{1 / 4}\left(1+O\left(q^{2}\right)\right) .
$$

On the right-hand side we have $i^{-1 / 4} \sqrt{2\left(q^{2}\right)^{1 / 4} / 2}\left(1+O\left(q^{2}\right)\right)$, which is the same. Hence we can simply square both sides and compare the results. For the left-hand side we have

$$
\begin{aligned}
\left(\frac{\theta_{1}^{\prime}\left(0, q^{2}\right) \theta_{3}\left(0, q^{2}\right)}{\theta_{1}^{\prime}(0, i q)}\right)^{2} & =\frac{\left(q^{2}\right)^{1 / 2}}{(i q)^{1 / 2}} \prod_{n=1}^{\infty} \frac{\left(1-q^{4 n}\right)^{8}}{\left(1-(-1)^{n} q^{2 n}\right)^{6}}\left(1+q^{4 n-2}\right)^{4} \\
& =\left(\frac{q}{i}\right)^{1 / 2} \prod_{n=1}^{\infty} \frac{\left(1-q^{4 n}\right)^{2}}{\left(1+q^{4 n-2}\right)^{2}} .
\end{aligned}
$$

For the right-hand side we have

$$
i^{-1 / 2} \frac{\theta_{2}\left(0, q^{2}\right) \theta_{4}\left(0, q^{2}\right)}{2}=\frac{2 i^{-1 / 2} q^{1 / 2}}{2} \prod_{n=1}^{\infty}\left(1-q^{4 n}\right)^{2}\left(1+q^{4 n}\right)^{2}\left(1-q^{4 n-2}\right)^{2}
$$

On comparison of the two sides we see that the result to be proved is given by

$$
1=\prod_{n=1}^{\infty}\left(1+q^{4 n-2}\right)^{2}\left(1+q^{4 n}\right)^{2}\left(1-q^{4 n-2}\right)^{2}
$$

or equivalently,

$$
1=\prod_{n=1}^{\infty}\left(1+q^{4 n}\right)\left(1-q^{8 n-4}\right)
$$

which is Eq. (29) with $q^{4}$ in place of $q$.

\section{References}

[1] F. W. J. Olver, D. W. Lozier, R. F. Boisvert, and C. W. Clark, NIST Handbook of Mathematical Functions, Cambridge University Press, New York, NY, 2010, print companion to [2].

[2] NIST Digital Library of Mathematical Functions, http://dlmf.nist.gov/, online companion to [1] with errata list and other updates.

About the author: Peter Walker resides in Cheltenham, UK. Previously, he was a faculty member at the University of Lancaster, UK, and the American University of Sharjah, UAE. He is the joint author, with William Reinhardt of the University of Washington, USA, of Chapters 20, 22, and 23 of Refs. [1] and [2]. The National Institute of Standards and Technology is an agency of the U.S. Department of Commerce. 